\title{
The impact of the rotation on the surface brightness of early-type stars
}

\author{
M. Challouf ${ }^{1,2}$, N. Nardetto ${ }^{1}$, A. Domiciano de Souza ${ }^{1}$, D. Mourard ${ }^{1}$, \\ H. Aroui ${ }^{2}$, P. Stee ${ }^{1}$ and A. Meilland ${ }^{1}$ \\ ${ }^{1}$ Laboratoire Lagrange, UMR7293, UNS/CNRS/OCA, 06300 Nice, France \\ email: mounir.challouf@oca.eu \\ ${ }^{2}$ Lab. Dyn. Moléculaire et Matériaux Photoniques, UR11ES03, UT/ESSTT, Tunis, Tunisie
}

\begin{abstract}
The surface brightness colors (SBC) relation is a very important tool to derive the distance of extragalactic eclipsing binaries. However, for early-type stars, this SBC relation is critically affected by the stellar environment (wind, circumstellar disk, etc...) and/or by the fast rotation. We calculated 6 models based on the code of Domiciano de Souza et al. (2012) considering different inclinations and rotational velocities. Using these results, we quantify for the first time the impact of the rotation on the SBC relation.
\end{abstract}

Keywords. stars: early-type, stars: rotation, techniques: interferometric, methods: numerical

\section{Introduction}

Detached eclipsing double-lined spectroscopic binaries offer a unique opportunity to measure directly, and very accurately the distance (Graczyk et al. 2011; Wyrzykowski et al. 2003; Macri et al. 2001) to nearby galaxies.

The early-type stars, very bright, are currently easily detected in the LMC (Pietrzyński et al. 2009; Pawlak et al. 2013) but there is no accurate SBCR available for these stars. Using the unique capabilities of the Visible spEctroGraph and polArimeter (VEGA) (Mourard et al. 2009) in this field of research, Challouf et al.(2014) have improved the surface brightness (with a $\sigma=0.17 \mathrm{mag}$ ) by determining the angular diameter of a dozen of OBA stars with a precision of $1.5 \%$.

An empirical SBCR is already well established for late-type stars (Di Benedetto 2005) coming from accurate determinations of stellar angular diameters by interferometry. But for early-type stars the interferometric study is a bit difficult because the majority of these stars have a stellar environment (wind, circumstellar disk, etc...) or they are fast rotators.

\section{Impact of the rotation}

For improving the surface brightness colors relationship for this type of star, we use numerical tool CHARRON (Code for High Angular Resolution of Rotating Objects in Nature) (Domiciano de Souza et al. 2012, 2002) code to model the rapid rotation and to better estimate the interferometric parameters. The stellar photospheric shape is given by the commonly adopted Roche approximation (rigid rotation and mass concentrated in the stellar center), which is well adapted for non-degenerate, fast rotating stars.

The effective temperature $T_{\text {eff }}$ at the surface for fast rotators is non uniform (dependend on the co-latitude $\theta$ ) due to the decreasing effective gravity $g_{\text {eff }}$ (gravitation plus centrifugal acceleration) from the poles to the equator (gravity darkening effect). We model the gravity darkening as a generalized form of the von Zeipel law (von Zeipel 
1924): $T_{\text {eff }}(\theta)=K g_{\text {eff }}^{\beta}(\theta)$ where $\beta$ is the gravity darkening coefficient, and $K$ is the proportionality constant between $T_{\text {eff }}$ and $g_{\text {eff }}$, which depends on the stellar physical parameters.

Once $T_{\text {eff }}(\theta)$ and $g_{\text {eff }}(\theta)$ are defined, we use the spectral synthesis code SYNSPEC (Hubeny \& Lanz 2011) and the ATLAS9 stellar atmosphere models (Kurucz 1979) to compute specific intensity maps of the star. The surface brightness relations can be directly obtained from these intensity maps and from the input parameters of the model, in particular equatorial radius and distance.

\begin{tabular}{lcccccc}
\hline model & M1 & M2 & M3 & M4 & M5 & M6 \\
\hline $\boldsymbol{V}-\boldsymbol{K}$ & -0.7 & -0.5 & -0.3 & 0 & 0.3 & 0.5 \\
$\boldsymbol{T}_{\text {eff }}[\mathbf{K}]$ & 23701 & 18418 & 13660 & 9794 & 8353 & 7822 \\
$\mathbf{l o g} \boldsymbol{g}[\mathbf{d e x}]$ & 4 & 3.5 & 4 & 4 & 4 & 4 \\
$\boldsymbol{M}\left[\boldsymbol{M}_{\odot}\right]$ & 13.61 & 5.59 & 4.11 & 2.52 & 2.17 & 1.99 \\
$\boldsymbol{R}\left[\boldsymbol{R}_{\odot}\right]$ & 10 & 5.01 & 2.88 & 2.39 & 2.23 & 1.99 \\
\hline
\end{tabular}

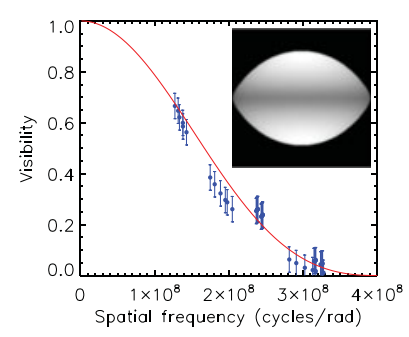

Table 1. Parameters of our sample based on Worthey \& Lee (2011)

Figure 1. Modeled intensity distributions and the fourier transform of the intensity map at $720 \mathrm{~nm}$ for $95 \%$ of $V_{c}$ and $i=90^{\circ}$

We used the intensity maps constructed by the CHARRON code (Fig. 1) describing the distribution of surface brightness of the stars from a number of parameters that depends on the type of objects studied (see Tab. 1). In a second step, we calculate the complex visibility from the Fourier transform (FT thereafter) of these maps in accordance with the theorem of Zernike-Van Cittert. We obtained thus the complex visibility. The module of the complex visibility provides the evolution of fringe contrast as a function of spatial frequency. In the case of a star deformed by the rotation, the diameter measurement must take into account the orientation of the interferometer in order to be interpreted correctly. In this case, the overall shape of the flattened star is determined by performing visibility measurements for different orientations of the interferometer (coverage of plane $(\mathrm{u}, \mathrm{v}))$ in order to probe different parts of the FT module. The visibility curves correspond to the component of the FT module along baselines that are then adjusted by a model of uniform disk (Fig. 1) using the similar configuration of the W1W2E2 and S2W2 VEGA/CHARA telescopes for 5 inclinations $\left(0^{\circ}, 25^{\circ}, 50^{\circ}, 75^{\circ}\right.$, and $\left.90^{\circ}\right)$ and 5 rotational velocity $(0.0 \mathrm{Vc}$, $0.25 \mathrm{Vc}, 0.50 \mathrm{Vc}, 0.75 \mathrm{Vc}$, and $0.95 \mathrm{Vc}$ ). With these diameters we can calculate the surface brightness for each star and by comparing the theoretical results and the simulation we estimate that the error caused by rotation is $0.12 \mathrm{mag}$ and the error due to the inclination is of $0.10 \mathrm{mag}$.

Acknowledgments: The research leading to these results has received funding from the European Community's Seventh Framework Programme under Grant Agreement 312430.

\section{References}

Di Benedetto, G. P. 2005, MNRAS 357, 174

Domiciano de Souza, A., Hadjara, M., Vakili, F., et al. 2012, A\&A 545, A130

Domiciano de Souza, A., Vakili, F., \& Jankov, S. e. a. 2002, A\&A 393, 345

Graczyk, D., Soszyński, I., Poleski, R., et al. 2011, AcA 61, 103

Hubeny, I. \& Lanz, T. 2011, Synspec, Astrophysics Source Code Library

Kurucz, R. L. 1979, ApJS 40, 1

Macri, L. M., Stanek, K. Z., Sasselov, D. D., \& et, a. 2001, AJ 121, 870 
Mourard, D., Clausse, J. M., Marcotto, A., et al. 2009, A\&A 508, 1073

Pawlak, M., Graczyk, D., Soszyński, I., et al. 2013, AcA 63, 323

Pietrzyński, G., Thompson, I. B., Graczyk, D., et al. 2009, ApJ 697, 862

von Zeipel, H. 1924, MNRAS 84, 665

Worthey, G. \& Lee, H.-c. 2011, ApJS 193, 1

Wyrzykowski, L., Udalski, A., Kubiak, M., et al. 2003, AcA 53, 1

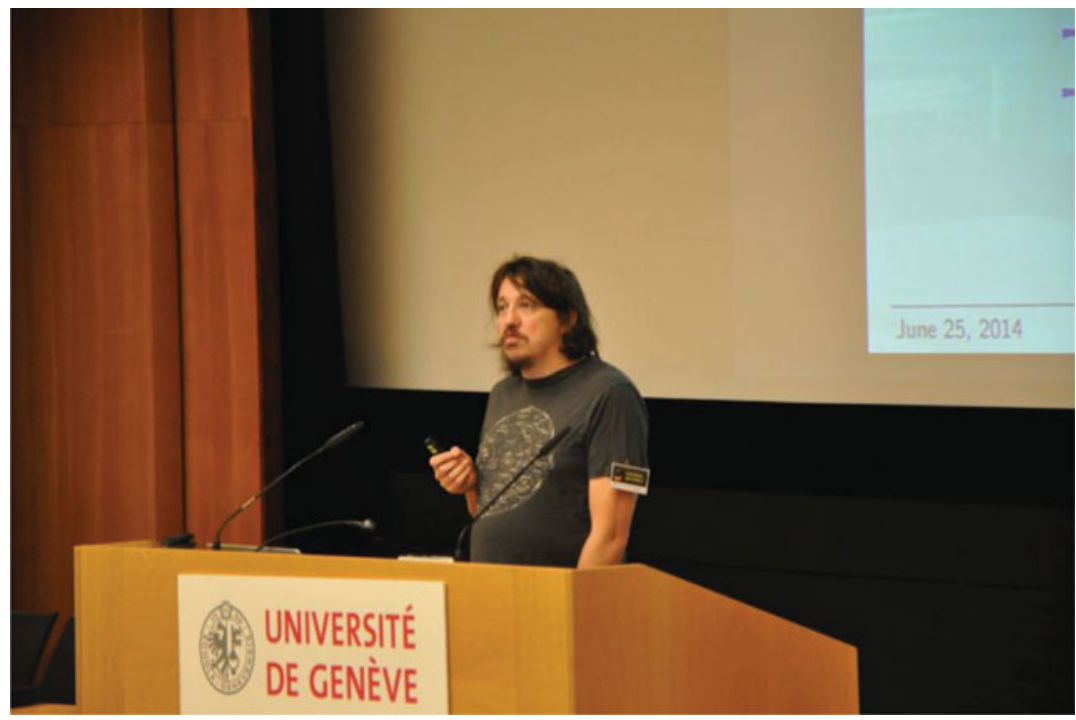

Thomas Rivinius

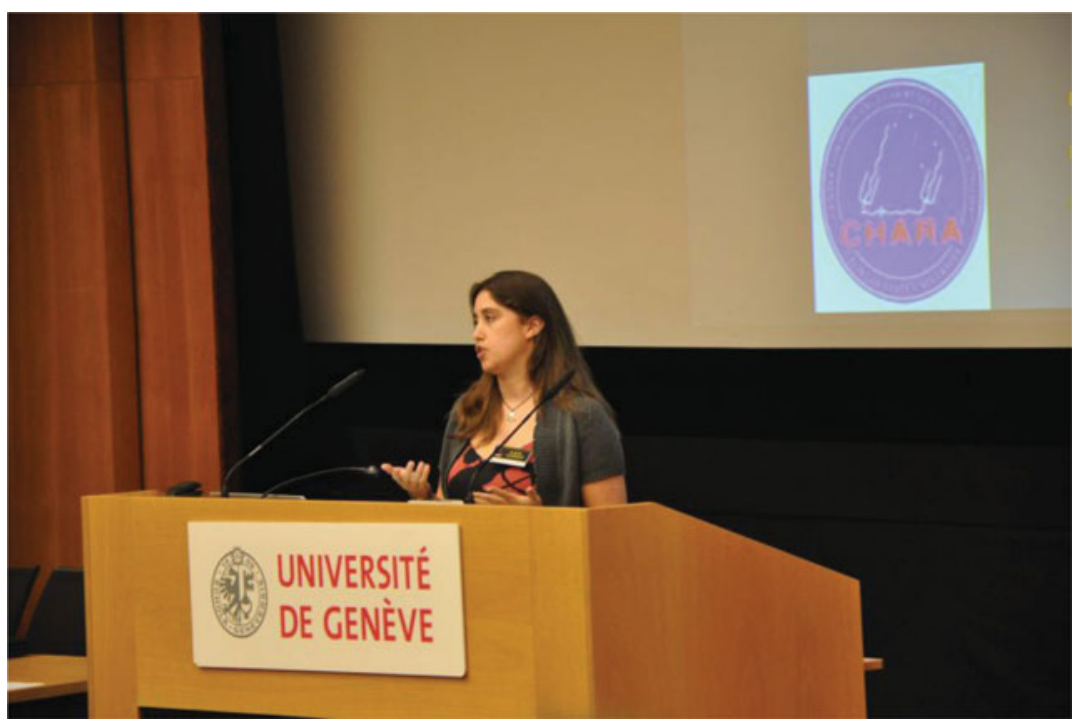

Katie Gordon 